\title{
Expert Mentoring: Assistente inteligente para esclarecimento de dúvidas sobre a determinação de evidências objetivas requeridas na avaliação MPS.BR
}

\author{
Lívia Maria Omena da Silva ${ }^{1}$, Rodrigo de Barros Paes ${ }^{1}$, Evandro de Barros Costa ${ }^{1}$ \\ ${ }^{1}$ Instituto de Computação - Universidade Federal de Alagoas (UFAL) \\ Campus A. C. Simões, Av. Lourival Melo Mota, s/n, Tabuleiro dos Martins \\ CEP 57072-970 - Maceió - AL - Brasil \\ \{livinhaomena, ebcosta\}@gmail.com, rodrigo@ic.ufal.br
}

\begin{abstract}
This paper presents an intelligent assistant, called Expert Mentoring, whose goal is to support managers responsible for processes GPR and GRE in the allocation of objective evidences, that are most appropriate for verification of the results contained in the spreadsheet of indicators used in evaluation MPS.BR. It is intended with this assistant, reproduce the behavior of the implementer to simulate the resolution of problems of interpretation of the results that occur while filling the spreadsheet. However, it is not intended to replace the implementer and, yes, support you in clarifying these doubts. It is hoped that this tool could be useful to organizations that will be submitted the assessment process.
\end{abstract}

Resumo. Este artigo apresenta um assistente inteligente, chamado Expert Mentoring, cujo objetivo é apoiar gerentes responsáveis pelos processos de GPR e GRE na alocação de evidências objetivas, que sejam mais adequadas para a comprovação dos resultados contidos na Planilha de Indicadores utilizada na avaliação MPS.BR. Pretende-se com este assistente, reproduzir o comportamento do implementador ao simular a resolução de problemas de interpretação dos resultados que ocorrem durante o preenchimento da planilha. Porém, não se pretende substituir o implementador e, sim, apoiá-lo no esclarecimento destas dúvidas. Espera-se que esta ferramenta possa ser útil às organizações que serão submetidas à avaliação de processos.

\section{Introdução}

De um modo geral, Sistemas Especialistas (SEs) têm sido abordados na literatura, cuja aplicabilidade se expande por diversas áreas do conhecimento. A literatura tem revelado SEs com objetivos específicos, de acordo com os trabalhos de: [Clancey and Letsinger 1982; Duda and Shortliffe 1983; Griesmer et al. 1984; Klatzky, Lederman and Metzger 1985; Astrom, Anton and Arzen 1986; Groothuis and Svensson 2000; Karabatak and Ince 2009], entre outros. Uma importante contribuição pode ser vista em [Liao 2005].

Em particular, este artigo pretende contribuir com o desenvolvimento do assistente inteligente, Expert Mentoring, na diminuição do esforço e tempo gastos pelos gerentes responsáveis pelos processos de Gerência de Projetos (GPR) e Gerência de Requisitos (GRE) durante o preenchimento da Planilha de Indicadores (PI), porque é 
considerável a quantidade de resultados esperados de processos e resultados de atributos de processos (RAPs) descritos nesta planilha. Além disso, pretende contribuir na aquisição de conhecimentos sólidos pelos gerentes sobre os processos de GPR e GRE, pois, é comum apresentarem dificuldades de interpretação dos resultados esperados de processos e RAPs. Também, contribuirá na ausência do implementador do modelo MPS, porque, em alguns casos, não dispõe de tempo suficiente para acompanhar o preenchimento ou revisão da PI, antes de submetê-la para avaliação informal ou formal.

O foco deste trabalho, inicialmente, está nos processos de GPR e GRE, por serem essenciais ao alcance de qualquer nível de maturidade [Chrissis, Konrad and Shrum 2006; Softex 2009a], e por exigirem um esforço menor para a definição da base de conhecimento do assistente inteligente proposto. É dada ênfase no Método de Avaliação para Melhoria de Processo do Software Brasileiro (MA-MPS) [Softex 2009b], que requer a apresentação de evidências objetivas, referentes ao nível de maturidade que será avaliado, com o objetivo de comprovar o atendimento aos resultados esperados de processos e RAPs descritos na PI [Idem 2009b]. Sendo esta PI, o ambiente onde essas evidências são apresentadas e, posteriormente, avaliadas pela equipe de avaliação de processos.

Além desta introdução, o artigo possui mais três seções. A seção seguinte apresenta uma caracterização do contexto de avaliação de processos ao qual este trabalho se refere. Na seção três, é proposto o assistente inteligente, Expert Mentoring, bem como, suas fases de desenvolvimento. Por fim, na seção quatro, algumas considerações sobre o trabalho são realizadas e os próximos passos descritos.

\section{Avaliação de Processos}

Em uma iniciativa de melhoria de processo de software mudanças são implementadas nos processos no intuito de introduzir melhorias [Olson, Humphrey and Kitson 1989]. Mas, implementar tal melhoria é uma atividade complexa e intensa de conhecimento [Montoni 2010 apud Minghui et al., 2004a]. Entretanto, milhares de organizações que desenvolvem software realizam iniciativas para melhorar seu desempenho. Assim, vários modelos de melhoria de software estão disponíveis [Stelzer and Mellis 1999], tais como: Capability Maturity Model - CMM [Paulk, Weber and Curtis 1995], Capability Maturity Model Integration for Development - CMMI-DEV [Carnegie Mellon 2006; Chrissis, Konrad and Shrum 2006], Padrões Internacionais: ISO/IEC 12207 [International Standard 2008] e ISO/IEC 15504-1 [International Standard 2004], Melhoria de Processo do Software Brasileiro - MPS.BR [Softex, 2009a], entre outros.

Independente da escolha, a implementação destes modelos revela resultados positivos e negativos. Sendo assim, tais resultados podem ser vistos nos trabalhos de: [Herbsleb et al. 1994; Loken and Skramstad 1996; Rodrigues 2009], que apresentam estudos empíricos sobre o sucesso e fracasso das iniciativas de melhoria de processos. No entanto, as avaliações de processo de software surgem como uma ferramenta importante e, comumente, usada para apontar o caminho, onde a organização precisa aplicar seus esforços em prol da melhoria dos processos. Para tal, a avaliação de processo é realizada por avaliadores que seguem critérios objetivos na identificação de pontos fortes e fracos dos processos de uma organização. Além disso, esta avaliação permite que um clima de mudança dentro da organização seja realizada [Dunaway and Masters 2001]. 
Por outro lado, avaliar os processos de software é uma atividade cara e num estado imaturo da organização, os resultados podem não ser identificados, pois, alguns processos podem levar tempo para serem executados e, no momento da avaliação, podem não estar desenvolvidos [Fayad and Laitinen 1997; El-Emam et al. 1998]. Além dos custos envolvidos, ao analisar a avaliação de processos proposta pelo MA-MPS, há casos em que o implementador não dispõe de tempo suficiente [Rocha et al. 2006] para acompanhar todo o preenchimento, ou revisão da PI antes de submetê-la à avaliação informal e/ou formal dos processos. Logo, erros de preenchimento da PI podem acontecer e comprometer o resultado da avaliação.

Como suporte à avaliação de processos de software, SEs são desenvolvidos no intuito de apoiar organizações no alcance da satisfação dos resultados estabelecidos por determinado modelo de melhoria de software. Desse modo, encontram-se na literatura alguns exemplos de SEs aplicados aos modelos: CMM, CMMI e ISO/IEC 15504, tais como: Virtual Auditor, que se propõe a determinar o grau de satisfação de cada KPA (Key Process Areas) do CMM, e a gerar um Plano de Ação que contenha os resultados do diagnóstico da organização, assim, apoiando o trabalho da auditoria interna ou externa [Herrera and Ramírez 2003]; Virtual Quality Editor, cujo objetivo é fornecer os resultados da avaliação dos modelos ISO/IEC 15504 e CMMI, e oferecer sugestões para as empresas para que a implementação dos modelos de qualidade seja bem sucedida [Eldrandaly 2008]; e o Diagnostic Intelligent Assistant, que sugere melhorias a partir de problemas identificados, e acompanha o progresso de cada processo proposto pelo CMMI [Adderley, Duggins and Tsui 2010]. No entanto, exemplos de SEs utilizados para apoiar à avaliação de processos proposta pelo modelo MPS não foram identificados na literatura durante o desenvolvimento desta pesquisa.

Diante disso, o assistente inteligente, Expert Mentoring, mostra-se interessante ao tentar minimizar os efeitos da falta de entendimento de gerentes responsáveis pelos processos de GPR e GRE sobre os resultados descritos na PI, bem como, da falta de tempo do implementador em acompanhá-los no preenchimento de toda esta planilha. Como a tarefa de preencher a PI é trabalhosa e requer bastante esforço, o Expert Mentoring, através de perguntas, pretende ajudar gerentes no entendimento sobre as características do indicador, que melhor satisfaz o resultado esperado e/ou RAP, à medida que dúvidas ocorrem. Por outro lado, sabe-se que certa dependência do implementador para a atualização da base de conhecimento do Expert Mentoring existirá, porque o conhecimento não é estático.

\section{Assistente Inteligente Proposto}

O Expert Mentoring é um assistente inteligente, que utiliza a abordagem de SEs, cujo objetivo é apoiar, através de perguntas, gerentes responsáveis pelos processos de GPR e GRE, e implementadores do modelo MPS na resolução de problemas de interpretação dos resultados esperados de processos e RAPs descritos na PI. Ao final destas perguntas, pretende-se que este assistente inteligente sugira nomes de indicadores diretos e indiretos, que sejam mais adequados para a comprovação desses resultados.

Para isso, o Expert Mentoring tenta reproduzir o comportamento do implementador do modelo MPS ao simular a resolução de problemas de interpretação de gerentes sobre os resultados esperados de processos e RAPs que ocorrem durante o preenchimento da PI. Porém, esta reprodução é bastante restrita por causa da dificuldade 
em captar todo o conhecimento deste implementador. Logo, é importante esclarecer que, não se pretende com o uso do Expert Mentoring substituir o implementador e, sim, apoiá-lo no esclarecimento de dúvidas que venham a ocorrer durante a preparação da PI. Portanto, pretende-se com o Expert Mentoring possibilitar o suporte em tempo integral aos gerentes responsáveis pelos processos de GPR e GRE, durante o preenchimento da PI, principalmente, na ausência do implementador.

A característica mais vantajosa do Expert Mentoring é a possibilidade de definir uma base de conhecimento baseada em regras de produção com a pretensão de representar a experiência do implementador do modelo MPS quanto à resolução de problemas de interpretação de gerentes sobre os resultados descritos na PI. Além disso, o Expert Mentoring mostra-se flexível na adaptação de sua base de conhecimento com novas soluções para problemas de interpretação de gerentes. Outras características podem ser mencionadas, tais como: explica como e por que foi sugerido o nome do indicador; e propõe ações de criação ou correção de indicadores diretos e indiretos para a solução do problema.

Contudo, o Expert Mentoring foi desenvolvido segundo as fases descritas no trabalho de [Castillo, Gutiérrez and Hadi 1997 apud Weiss, Kulikowski 1984], sendo estas: (i) Enunciar o problema; (ii) Encontrar especialistas humanos; (iii) Projetar o SE; (iv) Escolher uma ferramenta para desenvolvimento do SE; (v) Construir o protótipo; (vi) Testar o protótipo; (vii) Refinar e generalizar; e (viii) Manter e atualizar. A seguir, cada uma dessas fases será brevemente descrita, segundo o que foi feito durante o desenvolvimento do Expert Mentoring.

(i) Enunciar o problema: a concepção do problema ocorreu durante o preenchimento da PI pela investigadora desta pesquisa, que desempenhou o papel de Gerente de Projetos numa empresa alagoana, onde percebeu sua dificuldade em interpretar os resultados descritos na PI, mesmo tendo produzido algumas evidências e acompanhado a produção de outras; e de ter recebido treinamento prévio do implementador sobre o preenchimento da PI. Também, outra questão relevante de interpretação foi a confusão ocorrida na determinação de qual indicador é indireto para aquele que é direto. Já com relação ao implementador, este não dispunha de tempo suficiente para acompanhamento de todo preenchimento da PI, apesar de seu esforço em solucionar as dúvidas que ocorriam durante sua ausência da empresa.

(ii) Encontrar especialistas humanos: o papel de especialista humano foi desempenhado pela investigadora desta pesquisa, que adquiriu a competência necessária para o preenchimento da PI, pois, foi treinada por um implementador do modelo MPS e passou pelo processo de avaliação MPS.BR Nível F com êxito. Apesar das suas dificuldades durante o preenchimento da PI, pode-se afirmar que o processo permitiu sua aprendizagem. Também, esta investigadora desempenhou o papel de Engenheiro do Conhecimento ao traduzir o conhecimento sobre o preenchimento da PI numa linguagem que o assistente inteligente pudesse compreender. Por outro lado, a participação de um implementador experiente no fornecimento de conhecimento para $o$ Engenheiro do Conhecimento seria, indiscutivelmente, uma excelente opção, devido à sua experiência no modelo e em diversas organizações.

(iii) Projetar o SE: a partir da definição do problema, o Engenheiro do Conhecimento precisa realizar a modelagem da estrutura que definirá o Expert Mentoring, antes de implementá-la numa ferramenta, porque as características 
funcionais deste assistente inteligente influenciarão a escolha do ambiente de desenvolvimento. Inicialmente, foram definidos os requisitos que precisarão ser implementados para que o problema, aqui abordado, possa ser solucionado. Logo, os requisitos definidos foram: R01: Permitir a seleção do resultado esperado de processo ou RAP do nível G; R02: Apresentar uma descrição detalhada de cada resultado esperado de processo e RAP do nível G; R03: Realizar perguntas ao gerente sobre as características dos indicadores diretos e indiretos; R04: Sugerir indicadores diretos e indiretos, e/ou ações corretivas; e R05: Mostrar o caminho realizado pelo assistente inteligente para se chegar à conclusão. Em seguida, o processo de aquisição do conhecimento pelo Engenheiro de Conhecimento foi realizado através de consulta à literatura, pois, essa atitude é importante para que em futuras entrevistas com o Especialista, a conversa possa fluir no mesmo nível, onde uma linguagem técnica desconhecida poderá vir a impossibilitar o Engenheiro de Conhecimento na aquisição de determinadas informações. Também, a aquisição do conhecimento foi obtida através da experiência da investigadora desta pesquisa como Gerente de Projetos no preenchimento da PI utilizada numa avaliação de processos do Nível F.

(iv) Escolher uma ferramenta para desenvolvimento do SE: a ferramenta escolhida para o desenvolvimento do Expert Mentoring foi a Expert SINTA [Lia 199_?], porque satisfaz os critérios de gratuidade e facilidade de manipulação, aqui estabelecidos. Além disso, esta ferramenta utiliza um modelo de representação do conhecimento baseado em regras de produção com suporte ao tratamento de incerteza, através do uso de um motor de inferência compartilhado, da construção automática de telas e menus, e da utilização de explicações sobre o caminho percorrido para se chegar à conclusão.

(v) Construir o protótipo: a construção do Expert Mentoring foi realizada a partir da definição de sua base de conhecimento pelo Engenheiro do Conhecimento, que consistiu da criação de variáveis e seus respectivos valores, que permitem a organização da base; da definição de variáveis-objetivo, que controlarão o modo como o motor de inferência se comportará; da definição de regras de produção, onde o conjunto destas regras define a base de conhecimento; e da definição de interface, que estabelece a comunicação entre o Expert Mentoring e o usuário, através de menus de simples escolha, pois, foram consideradas variáveis do tipo uni-valoradas.

(vi) Testar o protótipo: o Expert Mentoring foi testado do seguinte modo: $1 . \mathrm{O}$ Engenheiro do Conhecimento, após a criação das regras, validou cada regra simulando o papel de gerente. Porém, sabe-se que esta validação realizada por quem criou, não é suficiente; 2. O Expert Mentoring foi submetido à avaliação de dois gerentes de uma empresa alagoana, que foi submetida à avaliação de processos do Nível F e obteve êxito em 2009. Um destes gerentes exerce o papel de Gerente de Projetos nessa empresa, onde também possui experiência como GQPP (Garantia da Qualidade do Produto e do Processo). Já o outro gerente desempenha o papel de Gerente de Métricas nessa empresa, além disso, é implementador certificado pela SOFTEX. Com isso, o teste foi realizado simulando o uso do Expert Mentoring. Logo, são destacados alguns exemplos de ponto forte: estratégia baseada em perguntas, que estimula o gerente a pensar nas características presentes no indicador para, depois, sugerir o nome deste indicador; ponto fraco: não amplamente utilizado num grande número de organizações submetidas à avaliação de processos; e oportunidades de melhoria: disponibilização de uma interface que facilite a consulta pelo usuário. 
(vii) Refinar e generalizar: nesta fase, buscou-se corrigir as falhas identificadas no Expert Mentoring, sendo que a principal recomendação foi corrigir a forma como a sugestão é apresentada pelo Expert Mentoring, pois, não demonstrava clareza no texto. Também, nesta fase são adicionadas novas possibilidades ao projeto inicial, só que neste contexto, nenhuma nova funcionalidade, além dos processos de GPR e GRE, foi adicionada à base de conhecimento do Expert Mentoring. Por outro lado, como trabalhos futuros, pretende-se ampliar esta base com os demais processos do MPS.BR.

(viii) Manter e atualizar: esta última fase do desenvolvimento do Expert Mentoring propõe que os gerentes relatem as falhas encontradas durante o uso deste assistente inteligente para que sejam ajustadas. Além disso, atualizações de novas regras podem ser feitas na base de conhecimento do Expert Mentoring. Em contrapartida, este assistente inteligente precisaria estar implantado numa organização, onde pudesse auxiliá-la na preparação da PI para a avaliação MPS.BR. Porém, no momento, não existem organizações alagoanas que estejam se preparando para esta avaliação. Já o deslocamento para outros estados torna-se inviável, devido à falta de recursos financeiros.

Apesar das características presentes no Expert Mentoring serem significativas, existem alguns fatores que limitam sua atual utilidade. Assim, eis algumas limitações baseadas no custo, controle e complexidade: (i) não amplamente utilizado ou testado num grande número de organizações, cujos processos serão avaliados; (ii) limitado a resolver problemas relativamente simples sobre os resultados esperados de processos e RAPs do nível G; (iii) o conhecimento está representado por meio de regras definidas, onde comparações são realizadas com casos semelhantes, caso contrário, não possui uma solução; (iv) possibilidade de erros na escolha da solução do problema pelo implementador do modelo MPS e/ou na tradução desta solução numa linguagem que o assistente inteligente possa compreender por parte do Engenheiro do Conhecimento; e (v) o assistente inteligente não é capaz de refinar sua base de conhecimento, sendo necessária a participação de um Engenheiro do Conhecimento no fornecimento de instruções sobre novas experiências.

\section{Considerações Finais e Próximos Passos}

$\mathrm{O}$ assistente inteligente, Expert Mentoring, foi apresentado neste artigo com o propósito de apoiar, através de perguntas, gerentes responsáveis pelos processos de GPR e GRE, e implementadores do modelo MPS na resolução de problemas de interpretação dos resultados esperados de processos e/ou RAPs descritos na PI. Ao final destas perguntas, pretende-se que este assistente inteligente sugira nomes de indicadores, que sejam mais adequados para a comprovação desses resultados. No entanto, esta assistência está limitada aos processos do nível $\mathrm{G}$, sendo considerado como próximos passos a ampliação da base de conhecimento com os demais níveis. Já a participação de implementadores experientes no modelo será viabilizada para a construção de regras de produção mais confiáveis, visando à implantação do Expert Mentoring nas organizações. Ressalta-se que este assistente inteligente está em fase de validação da base de conhecimento, onde, ainda, não está disponível para visualização pela comunidade.

\section{Agradecimentos}

Agradeço à FAPEAL pelo apoio financeiro que viabilizou esta pesquisa. 


\section{Referências}

Adderley, T., Duggins, S. and Tsui, F. (2010) "An examination of a rule-based expert system to aid in the implementation of the CMMI framework".

Astrom, K. J., Anton, J. J. and Arzen, K. E. (1986) "Expert Control”, In: Automatica, v.22, n.3, p.277-286.

Carnegie Mellon (2006) "CMMI for Development" (v1.2), Software Engineering Institute.

Castillo, E., Gutiérrez, J. M. and Hadi, Ali S. (1997) "Expert systems and probabilistic network models", New York: Springer, cap. 1 e 2, p.1-65.

Clancey, William J. and Letsinger, Reed (1982) "Neomycin: reconfiguring a rulebased expert system for application to teaching”, Computer Science Department Stanford University, Stanford.

Chrissis, M. B., Konrad, M. and Shrum, S. (2006) "CMMI (Second Edition): Guidelines for Process Integration and Product Improvement", Addison Wesley Professional.

Duda, R. O. and Shortliffe, E. H. (1983) “Expert Systems Research”, In: Science, v.220, n.4594.

Dunaway, D. K. and Masters, S. (2001) " $\mathrm{CMM}^{\mathrm{R}}$-Based Appraisal for Internal Process Improvement (CBA IPI) Version 1.2 Method Description”, Carnegie Mellon.

Eldrandaly, K. (2008) "A Knowledge-Based Advisory System for Software Quality Assurance", In: The International Arab Journal of Information Technology, v.5, n.3.

El-Emam, K., et al. (1998) "Cost Implications of Interrater Agreement for Software Process Assessments", In: International Software Engineering Research Network Technical Report ISERN-98-14, v.4.

Fayad, M. E. and Laitinen, M. (1997) "Process Assessment Considered Wasteful”, In: Communications of the ACM, v.40, n.11, p.125-128.

Griesmer, J. H., et al. (1984) "YEWMVS: A Continuous Real Time Expert System", AAAI-84 Proceedings.

Groothuis, M. M. and Svensson, J. (2000) "Expert system support and juridical quality", In: Legal Knowledge and Information Systems, The Thirteenth Annual Conference.

Herbsleb, J., et al. (1994) "Benefits of CMM-Based Software Process Improvement: Initial Results", Empirical Methods, Carnegie Mellon University.

Herrera, E. M. and Ramírez, R. A. T. (2003) "A Methodology for Self-Diagnosis for Software Quality Assurance in Small and Medium-Sized Industries in Latin America".

International Standard (2008) "ISO/IEC 12207: Systems and software engineering software life cycle processes".

International Standard (2004) "ISO/IEC 15504-1: Information technology - process assessment".

Karabatak, M. and Ince, M. C. (2009) "An expert system for detection of breast cancer based on association rules and neural network", In: Expert Systems with applications. 
Klatzky, R. L., Lederman, S. J. and Metzger, V. A. (1985) "Identifying objects by touch: An expert system", In: Perception \& Psychophysics.

LIA - Laboratório de Inteligência Artificial. (199_?) "Expert Sinta versão 1.1: uma ferramenta visual para criação de sistemas especialistas", Manual do Usuário.

Liao, Shu-Hsien (2005) "Expert system methodologies and applications: a decade review from 1995 to 2004", In: Expert Systems with Applications.

Loken, C. B. and Skramstad, T. (1996) "ISO 9000 certification: Experiences from Europe", In: Product Assurance Symposium and Software Product Assurance Workshop, Proceedings of the meetings held, European Space Agency, p.295-301.

Montoni, M. A. (2010) "Uma investigação sobre os fatores críticos de sucesso em iniciativas de melhoria de processos de software", Universidade Federal do Rio de Janeiro (Tese de Doutorado), p.328, Rio de Janeiro.

Olson, T. G., Humphrey, W. S. and Kitson, D. (1989) "Conducting SEI-Assisted Software Process Assessments", Technical Report, CMU/SEI-89-TR-7, ESD-89-TR7. p.1-62. Pittsburgh.

Paulk, M. C., Weber, C. V. and Curtis, M. C. (1995) "The Capability Maturity Model ${ }^{\mathrm{sm}}$ for Software: Guidelines for Improving the Software", Software Engineering Institute.

Rocha, Ana R. C., et al. (2006) "Dificuldade e Fatores de Sucesso na Implementação de Processos de Software Utilizando o MR-MPS e o CMMI", I Workshop de Implementadores (W2-MPS.BR).

Rodrigues, J. F. (2009) "Avaliação da implantação do MPS.BR: um estudo empírico sobre benefícios, dificuldades e fatores de sucesso", Universidade Metodista de Piracicaba (Dissertação de Mestrado).

Softex (2009a) "MPS.BR - Melhoria de Processo do Software Brasileiro, Guia Geral (v1.2)”, SOFTEX - Associação para Promoção da Excelência do Software Brasileiro.

Softex (2009b) "MPS.BR - Melhoria de Processo do Software Brasileiro, Guia de Avaliação", SOFTEX - Associação para Promoção da Excelência do Software Brasileiro.

Stelzer, Dirk and Mellis, Werner (1999) "Success Factors of Organizational Change in Software Process Improvement", Software Process Improvement and Practice, v.4, p.1-34, Issue 4, Germany. 